\title{
Prevalence of food addiction among Iranian children and adolescents: Associations with sociodemographic and anthropometric indices
}

\author{
Mahshid Naghashpour ${ }^{1,2}$, Rouhollah Rouhandeh ${ }^{1}$, Marzieh Karbalaipour ${ }^{3,4}$, Mahsa Miryan ${ }^{3,4 *}$ \\ Received: 26 Aug 2017 \\ Published: 8 Feb 2018
}

\begin{abstract}
Background: Food addiction has been defined as consuming palatable foods leading to addictive-like behaviors. Little studies have assayed food addiction in children and adolescents. Thus, we aimed at identifying the prevalence of food addiction and the relationship between food addiction, sociodemographic and anthropometric indicators among children and adolescents in southwestern Iran.

Methods: This cross-sectional study was conducted on 222 elementary school students aged 7 to 13 years in Ahvaz, Iran, using a random sampling method. Sociodemographic and anthropometric indicators were obtained. The 25 -item child version of the Yale Food Addiction Scale (YFAS-C) was applied to provide food addiction diagnosis and symptoms. A nonparametric analysis was used for data analysis.

Results: The prevalence of food addiction was $17.3 \%$. Also, the most common symptoms associated with food addiction were (1) inability to cut down, (2) withdrawal, and (3) tolerance. The students with food addiction diagnosis were older than the undiagnosed $(\mathrm{p}=0.04)$. The males and students older than 8 years showed a higher food addiction score than females and students under 8 years $(\mathrm{p}<0.05)$. In females, significant positive correlations were found between anthropometric indicators, including body mass index and body mass index $\mathrm{z}$-score, with food addiction score $(\mathrm{p}<0.01)$.

Conclusion: Food addiction diagnosis was more prevalent in males and students over 8 years. Females with higher YFAS-C scores had elevated body mass index z-scores, suggesting that food addiction may be a remarkable problem in childhood and early adolescence and may also be related to a risk of overweight/obesity in Iranian students.
\end{abstract}

Keywords: Food addiction, Body mass index, Demography, Student, Iran

Copyright $\odot$ Iran University of Medical Sciences

Cite this article as: Naghashpour M, Rouhandeh R, Karbalaipour M, Miryan M. Prevalence of food addiction among Iranian children and adolescents: Associations with sociodemographic and anthropometric indices. Med J Islam Repub Iran. 2018(8 Feb);32:8. https://doi.org/10.14196/mjiri.32.8

\section{Introduction}

While there is no agreement upon definition of addictivelike eating in humans (1), clinical data suggest that consuming palatable foods may lead to addictive-like behaviors in some individuals. Based on this observation, interest in the phenomenon of food addiction has increased substantially in scientific research (2). Food addiction is defined as a persistent course to crave for food resulting from hedonic factors. Animal models of food addiction find that rats given access to high-fat, high-sugar, and processed foods exhibit

Corresponding author: Mahsa Miryan, miryanm2014@gmail.com

1. Abadan School of Medical Sciences, Abadan, Iran

2. Nutrition and Metabolic Diseases Research Center, Ahvaz Jundishapur University of Medical Sciences, Ahvaz, Iran

3. Student Research Committee, Ahvaz Jundishapur University of Medical Sciences, Ahvaz, Iran

4. Department of Nutrition, School of Paramedicine, Ahvaz Jundishapur University of Medical Sciences, Ahvaz, Iran classic behavioral and biological signs of addiction (e.g. withdrawal, tolerance, and dopaminergic downgrading) (3, 4). In the general population of adults, food addiction has been associated with hormonal (e.g. a higher concentration of prolactin and lower concentration of tumor necrosis factor- $\alpha$ (TNF- $\alpha$ ), amylin, and follicle-stimulating hormone (FSH) in food-addicted overweight/obese individuals compared to the non-food-addicted ones) (5), biological (e.g. reward dysfunction) $(6,7)$, mental (e.g. depression, anxiety,

$\uparrow$ What is "already known" in this topic:

Food addiction prevalence is $17.3 \%$ in a population of Iranian children and adolescents. "Inability to cut down" is the most common contributor to their clinical eating problems. Food addiction is related to demographic and anthropometric indices related to the risk of overweight and obesity among this age group.

\section{$\rightarrow$ What this article adds:}

Age over 8 years and male gender were the most important risk factors for food addiction in Iranian elementary school students. Moreover, Iranian female students were at a higher risk for obesity and overweight with "food addiction" than males. 
and stress) $(8,9)$, and behavioral (e.g. cravings, impulsivity) (10-12) factors implicated in addictive disorders.

Despite an increasing evidence on the prevalence of food addiction in adults $(6,7,11,13-23)$ and the publication of a systematic review in this context (24), little studies have assayed the prevalence of food addiction in children and adolescents $(25,26)$. Also, to date, sociodemographic indicators related to food addiction in children and adolescents have not been investigated. Moreover, no information is available on the prevalence of food addiction among Iranian children and adolescents. The issue needs to be further explored in larger cross-sectional surveys in different geographical areas, with different cultures, customs, and ethnicities and various foods. Moreover, future studies should consider such variables as anthropometric and demographic indicators to determine the relationships with food addiction status in children and adolescents (25). Based on the addiction literature, children are more vulnerable to the negative effects of addictive substances than adults (27). Thus, if certain foods are addictive, children might be at more risk of addictive-like eating behavior compared to adults (28).

Several studies have also examined the possibility of a causal relationship between food addiction and the subsequent obesity epidemic (1). The prevalence of food addiction is increasing in obese patients (29) and encompasses different age groups $(26,30)$. In adults $(8,9,13)$ and children (25), food addiction is associated with elevated body mass index (BMI) and visceral adiposity (31). Whereas the initial drugs abuse (e.g. alcohol and cannabis) typically occurs during adolescence and early adulthood (32), consumption of potentially addictive foods (e.g. candy, cake, sugar-sweetened beverages) is more likely to occur during early childhood $(33,34)$. The repeated consumption of highly palatable foods and the subsequent dependence early in development may be a cause of obesity epidemic in childhood $(1,35)$. Also, dietary habits originate from childhood and continue to adulthood. Accordingly, the evaluation of addictive-like eating in children and adolescents may cover the gaps on prevention and treatment of obesity in children and adolescents (36).

According to our knowledge, evidence supporting the concept of food addiction among Iranian adults is limited (18). The relationship between food addiction, demographic indices, and obesity has not been studied extensively. Thus, the present study was performed to detect the frequency of food addiction and evaluate the relationship between food addiction, sociodemographic and anthropometric indicators among Iranian elementary school students.

\section{Methods}

\section{Study setting}

We conducted a cross-sectional study on 3908 students (1868 males and 2040 females) aged 7 to 13 years studying in elementary schools of Ahvaz, the capital city of Khuzestan province, Iran, over 3 months (January to March 2017). Ahvaz organization of education and training consists of 4 educational districts including public and nonprofit elementary schools. A two-stage random sampling method was conducted to yield a sample generalizable to all elementary students in Ahvaz. In the first stage, 8 schools ( 4 girls' and 4 boys' schools) were selected randomly out of 311 public elementary schools (151 girls' and 160 boys' schools) in 4 educational districts in Ahvaz (one girls' school and one boys' school from each educational district). Since the unit of randomization was the classes of schools, in the second stage, one class within each school was randomly selected. Grades of classes (first to sixth grades) were randomly selected. According to a similar study, the prevalence of food addiction among children was $38.6 \%$ (25). Considering the $95 \%$ confidence interval and

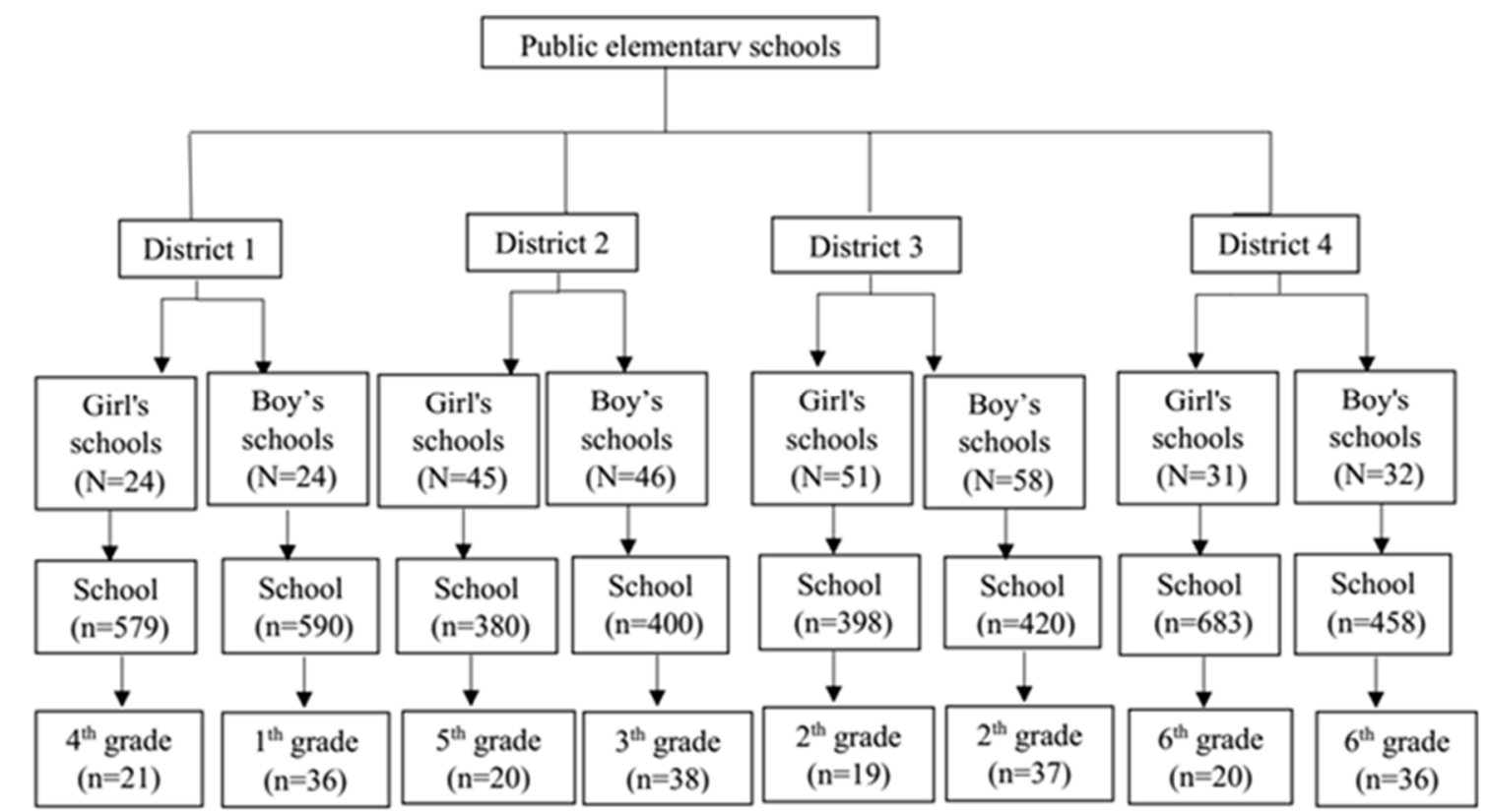

Fig. 1. Recruitment and enrollment of study participants, $\mathrm{N}=$ the number of schools in each district, $\mathrm{n}=$ the number of students in each selected school and class 
based on the formula given in the following equation, the minimum number that could be calculated for measuring the prevalence was 177 students with $25 \%$ chance of losing samples, so the sample size was estimated 222 elementary school children.

$$
n=\frac{z^{2} \times(p * 1-p)}{\delta^{2}}
$$

$\mathrm{Z}=\mathrm{Z}$ value (e.g. 1.645 for confidence interval), $\mathrm{p}=\mathrm{prev}-$ alence ratio. $\delta=$ Error margin $=0.02$.

Finally, on average, 28 students were enrolled in the study in each cluster (classes of schools) from different educational grades (Fig. 1 and Table 1).

Initially, an introduction letter was sent to authorities of schools to cooperate with researchers. They were also asked to provide the demographic information of students to the researchers.

\section{Measures}

Sociodemographic indicators, which included information on sex, age, parents' occupation, and parental education level, were obtained from the recorded information in school files of each student to evaluate the socioeconomic status. Anthropometric indices including weight and height were also measured, which were converted into BMI using standardized equations. BMI values were transformed into BMI z-scores, a measure of relative weight adjusted for the child's age and sex. Based on the World Health Organization (WHO) guidelines of fixed exclusion, BMI z-scores lower than -4.0 and higher than +5.0 are considered to be biologically implausible values and should be excluded from the analysis (World Health Organization (WHO), 1995). Typically, these values (outliers) are the result of misreporting rather than true growth extreme. Children's BMI z-scores were categorized according to the WHO BMI-for-age (5-19 years) cut-off points (37). The students were categorized according to their BMI z-scores as follow: underweight (BMI z-score $<-2 \mathrm{SD}$ ), healthy weight $(-1 \leq$ BMI $\mathrm{z}$-score $\leq 0)$, overweight $(1 \leq \mathrm{BMI} \mathrm{z}$ score $<2)$, obese $(+2 \geq$ BMI $z$-score $>3)$, and morbidly obese (BMI z-score $\geq+3$ ) (36).

\section{Questionnaire translation}

The Persian version of the child version of the YFAS (YFAS-C) (25) was applied to evaluate food dependence. The YFAS-C questionnaire was translated from English into Persian by 2 competent expert translators. Then, the translations were integrated by agreement between translators and researchers. Also, the dietary cultural adaptation was done to ensure that the translated questionnaire was easy and understandable for Persian language students. During dietary cultural adaptation, 5 food items of original YFAS-C were deleted and 5 new items were added. In a pilot study, the validity of the Persian version was evaluated by distributing its prefinal version randomly to 20 native Persian speaking students, and its comprehensibility was assessed. Necessary changes were exerted to make the questionnaire understandable. Also, 5 nutritionists (full professors of medical universities) revised the translated questionnaire. Finally, the revised YFAS-C was used in a survey of 222 elementary students.

YFAS-C has 25 items, which was developed by Gerardhard et al. (25). Items were computed under criteria adopted from the symptoms for substance dependence in the fourth revision of the Diagnostic and Statistical Manual of Mental Disorders (DSM-IV). The diagnostic criteria for substance dependence according to the DSM-IV are as follow: (1) tolerance, defined as "consuming increasing amounts of a substance to reach the same effects or feeling reduced effects with continuous use of the same quantities"; (2) withdrawal symptoms "when the substance is not consumed or using the substance to avoid withdrawal symptoms"; (3) loss of control defined as "consuming the substance in larger quantities or over a longer period than intended"; (4) inability to cut down, defined as "a persistent desire or unsuccessful efforts to cut down substance use"; (5) much time/activity to obtain, use, or repair, that is, " an increasing efforts to gain or use the substance or its effects recovery"; (6) giving up activities defined as "reduction of social, occupational, or recreational activities because of substance use"; and (7) use, despite of negative consequences, that is, " the use of the substance despite persistent physical or psychological problems caused or exacerbated by the substance" (38). Finally, these criteria provided a food addiction diagnosis. In our study, food addiction was classified as "mild", "moderate" or "severe". A "mild diagnosis" score is assumed when 3 or more criteria are reported, a "moderate" score is gained when 4 to 5 criteria are present, and "severe" is when 6 or more criteria are discerned (9). The YFAS-C scale yields 2 outcome variables. The first is a continuous measure of endorsed symptoms, which is the total number of substance dependence symptoms reported by the respondent over the past year (ranging from 0 to 7 ). The second is a dichotomous measure, presenting a 'diagnosis' of food addiction when the respondents experience 3 or more symptoms over the past year accompanied by the "clinically significant impairment/distress" criterion (9). In the present study, students at forth to sixth grades completed the YFAS-C as self-report and parents of first to third grade students were asked to provide data on their children's addictive-like eating behavior. As for the YFAS-C in this study, the word 'I' was replaced by 'your child' with pronouns changed from reporting on oneself to reporting on one's child. For the purpose of this study, both symptom and diagnosis scores were used.

\section{Ethical considerations}

Before collecting the demographic and food addiction data, the necessary permissions from the school authorities and/or parents of all participants were obtained by asking them to complete an informed consent, which was in agreement with the provisions of the Declaration of Helsinki. Also, they were reassured that personal information of students would be kept confidential. Study protocol was reviewed and given final approval from the Student Research Committee, office of the Vice-Chancellor for Research, Ahvaz Jundishapur University of Medical Sciences (Grant number: 94S119). Moreover, the ethics committee of Ahvaz Jundishapur University of Medical Sciences (AJUMS) 
approved this study (Ethics Committee Code: IR. AJUMS.REC.1395.512).

\section{Statistical analysis}

Firstly, the original sample of $(n=222)$ students was categorized in to 2 groups according to their food addiction diagnosis: students meeting food addiction diagnostic criteria on the YFAS-C ( $<3$ criteria fulfilled; named diagnosed $\mathrm{n}=171$ ), and those students with food addiction diagnosis on the YFAS-C ( $\geq 3$ criteria fulfilled and clinically significant impairment/distress; named undiagnosed; $n=37$ ). In the second classification, the students diagnosed with food addiction were classified into "mild", "moderate", or "severe" categories.

All analyses were performed using SPSS Statistics (Version 21, IBM, Armonk, NY, 2010). Preliminary descriptive analyses were performed on all data. Quantitative variables including age, anthropometric indicators, and food addiction score on continuous version of scale were not normally distributed (based on the Shapiro-Wilk statistic, skewness, and kurtosis). Therefore, the analyses were performed using nonparametric procedures. The Mann-Whitney U-test was conducted to compare differences between the 2 independent groups including students with food addiction di- agnosis and those undiagnosed on the continuous dependent variables including food addiction score on the continuous version of the scale, age, and anthropometric indicators. Also, Kruskal-Wallis test was utilized to find statistically significant differences between student groups with different food addiction severity as an independent variable on continuous dependent variables including age and anthropometric indicators. Moreover, chi-square test was used to compare categorical data including frequency of symptoms on YFAS-C and food addiction severity between the students with and without food addiction diagnosis. Besides, the Spearman's rho coefficient was used for correlations. Correlation coefficients were computed between the food addiction score and the anthropometric indicators (2-tailed tests).

\section{Results}

\section{Characteristics of the study population}

Sociodemographic and anthropometric characteristics of the participants who completed YFAS-C are presented in Table 1. A total of 222 individuals were recruited for the study. The age of the students $(65.9 \%$ male $)$ ranged from 6 to 14 years $(9.6 \pm 1.7$ years). Most of the students had retired fathers or fathers who were workers and employees, with no college education. Also, more students had non-

Table 1. Sample size, sociodemographics, and anthropometric characteristics among Iranian elementary school students ${ }^{\dagger}$

\begin{tabular}{|c|c|}
\hline Variable & Baseline characteristics \\
\hline No. of classes & 8 \\
\hline \multicolumn{2}{|l|}{ No. of students by grade (\%) } \\
\hline $1^{\text {th }}$ & $25(11.3)$ \\
\hline $2^{\text {th }}$ & $44(19.8)$ \\
\hline $3^{\text {th }}$ & $28(12.6)$ \\
\hline $4^{\text {th }}$ & $38(17.1)$ \\
\hline $5^{\text {th }}$ & $65(29.3)$ \\
\hline $6^{\text {th }}$ & $22(9.9)$ \\
\hline \multicolumn{2}{|l|}{ Demographic characteristics } \\
\hline Age (yr) & $9.6 \pm 1.7$ \\
\hline \multicolumn{2}{|l|}{ Age category $(\%)^{\varphi}$} \\
\hline$<8 \mathrm{yr}$ & $69(30.9)$ \\
\hline$>8 \mathrm{yr}$ & $154(69.1)$ \\
\hline Male sexuality $(\%)$ & $147(65.9)$ \\
\hline \multicolumn{2}{|l|}{ Socioeconomic status $(\%)^{\ddagger}$} \\
\hline \multicolumn{2}{|l|}{ Paternal occupation } \\
\hline Worker, employee, retired & $120(55.6)$ \\
\hline Practitioner (medical physician, lower, etc.) and self-employment & $96(44.4)$ \\
\hline \multicolumn{2}{|l|}{ Maternal occupation } \\
\hline Employed & $38(17.6)$ \\
\hline Non-employed (housewife and retired) & $178(82.4)$ \\
\hline \multicolumn{2}{|l|}{ Paternal education level } \\
\hline College education & $107(48.4)$ \\
\hline Non-college education (Diploma and less) & $114(51.6)$ \\
\hline \multicolumn{2}{|l|}{ Maternal education level } \\
\hline College education & $83(37.7)$ \\
\hline Non-college education (Diploma and less) & $137(62.3)$ \\
\hline \multicolumn{2}{|l|}{ Anthropometric measurements } \\
\hline Height $(\mathrm{cm})$ & $138.1 \pm 11.6$ \\
\hline Weight (kg) & $36.5 \pm 12.8$ \\
\hline Body-mass index (BMI)* & $18.6 \pm 4.3$ \\
\hline BMI z-score & $0.46 \pm 1.6$ \\
\hline \multicolumn{2}{|l|}{ BMI z-score category ${ }^{\S}(\%)$} \\
\hline Underweight & $33(14.9)$ \\
\hline Healthy weight & $72(32.4)$ \\
\hline Overweight & $48(21.6)$ \\
\hline Obese & $49(22.1)$ \\
\hline Morbidly obese & $20(9)$ \\
\hline
\end{tabular}

$\dagger$ Plus-minus values are means \pm SD. ${ }^{\varphi}$ Data on age category were missing for 4 children. $₫$ Data on each paternal and maternal occupation were missing for 11 children, data on paternal education level were missing for 6 children, and data on maternal education were missing for 7 children. ${ }^{*}$ Body-mass index is the weight in kilograms divided by the square of the height in meters. § WHO BMI-for-age (5-19 years) -cut-offs (37). 
employed mothers with no college education, which was indicative of a moderate socioeconomic status. The mean BMI of the students was normal $(18.6 \pm 4.3)$. After BMI zscores classification, most of the students were at a healthy weight. There were no significant differences between males and females in BMI, weight, BMI z-score, and BMI z-score category.

\section{Student's food addiction frequencies}

Table 2 demonstrates the comparison of symptom scores, frequency of symptoms, and food addiction score on the continuous version of the YFAS-C scale for students with and without food addiction diagnosis. A total of 36 students (17.3\%; $17.8 .4 \%$ of males, $16.4 \%$ of females) met the food addiction diagnostic criteria. The mean and standard deviation $($ Mean \pm SD) of food addiction symptom score was $4.6 \pm 1.2$ for the diagnosed and $2.3 \pm 1.2$ for the undiagnosed children and adolescents, respectively. The most prevalent symptoms associated with food addiction included "inability to cut down", "withdrawal", "tolerance", "giving up activities", and "much time/activity to obtain, use, and recover", which were reported by $91.7 \%, 80.6 \%, 72.2 \%$, $69.4 \%$, and $63.9 \%$ of the students in whom food addiction was detected as a contributor to their eating problems. Moreover, in an additional analysis among students with food addiction diagnosis, $47.2 \%(\mathrm{n}=17)$ and $52.8 \%(\mathrm{n}=19)$ had moderate and severe food addiction, respectively. None of the students with food addiction diagnosis had mild food addiction.

\section{Children food addiction and sociodemographics}

Non-parametric tests were used to evaluate the relationship between sociodemographic and anthropometric variables with food addiction diagnosis status and category of food addiction severity. Mann-Whitney $U$ test showed a significant difference in age among the students with food addiction diagnosis and undiagnosed ones. Moreover, chisquare test results showed a significant difference between age category and food addiction diagnosis. The students with food addiction diagnosis had a significantly higher mean rank of age than undiagnosed students $(p<0.05)$. Also, students aged over 8 years showed a more prevalence of food addiction than students aged under 8 years $(p<0.05$,
Table 3). Furthermore, Mann-Whitney U test presented statistically significant differences in food addiction score on the continuous version of the scale between male and female students. The male students had a significantly higher food addiction score on the continuous version of theYFAS-C than females $(p<0.05$, Table 4$)$. Also, the Mann-Whitney U test was applied to compare children aged under 8 years and those aged over 8 years based on food addiction scores on the continuous version of the YFAS-C. The results indicated that children aged over 8 years had a significantly higher food addiction score than children aged under 8 years $(p<0.05$, Table 4$)$. After division by sex, this difference persisted in males, but not in females; outputs of the Mann-Whitney $U$ test provided the following values: Mann-Whitney $U=1012.5, p<0.05$, with a mean rank food addiction score of 47.3 for male students aged less than 8 years and 61.8 for male students aged over 8 years.

However, Kruskal-Wallis $\mathrm{H}$ test showed no statistically significant differences in age between the students with different categories of food addiction severity $(p>0.05)$. Also, chi- square test results showed no significant difference in sex and socioeconomic status variables between students with and without food addiction diagnosis and category of food addiction severity.

\section{Children food addiction and anthropometrics}

Spearman partial correlation coefficients were applied to evaluate the relationship between the food addiction score on the continuous version of the YFAS-C scale and anthropometric indicators in the entire sample and in both sexes. All measured anthropometric indicators had significant positive correlations with food addiction score in the entire sample. In female, but not in male students, a significant positive correlation was found between food addiction score, BMI, and BMI z-score. Also, weight and height had a positive correlation with food addiction score in male students. Moreover, there was a significant positive correlation between food addiction score and weight in the entire sample and both sexes, which remained significant when it was controlled for possible confounding factors including age (Table 5).

Table 2. Comparison of symptom scores, frequency of symptoms, food addiction score, and category of food addiction severity between Iranian elementary school students with and without food addiction"

\begin{tabular}{|c|c|c|c|c|}
\hline Food addiction variables & $\begin{array}{c}\text { Undiagnosed } \\
(\mathrm{n}=172)\end{array}$ & $\begin{array}{c}\text { Diagnosed }^{\dagger} \\
(\mathrm{n}=37)\end{array}$ & $\begin{array}{c}\text { Test statistic } \\
(\chi 2 / \text { Mann-Whitney } U)\end{array}$ & $\mathrm{p}$ \\
\hline \multicolumn{5}{|l|}{$\overline{\text { Food addiction symptoms on YFAS-C }{ }^{\ddagger}}$} \\
\hline Loss of control ${ }^{\phi}$ & $12(7.87 .2)$ & $14(38.9)$ & 26.7 & $<0.001$ \\
\hline Inability to cut down ${ }^{6}$ & $123(80.4)$ & $33(91.7)$ & 2.6 & $>0.05$ \\
\hline Much time/activity to obtain, use, recover ${ }^{\dagger \dagger}$ & $48(28.4)$ & $23(63.9)$ & 16.5 & $<0.001$ \\
\hline Given up activities $\mho$ & $40(24.2)$ & $25(69.4)$ & 27.6 & $<0.001$ \\
\hline Use, despite negative consequences & $57(33.1)$ & $17(47.2)$ & 2.6 & $>0.05$ \\
\hline Tolerance ${ }^{\S}$ & $62(37.3)$ & $26(72.2)$ & 14.6 & $<0.001$ \\
\hline Withdrawal & $37(22.3)$ & $29(80.6)$ & 45.7 & $<0.001$ \\
\hline Continuous version of the scale $e^{\epsilon}$ & 74.4 & 146.2 & 472.5 & $<0.001$ \\
\hline
\end{tabular}

The results were assessed using chi-square test (shown as $\mathrm{n}(\%)$ ) and Mann-Whitney $\mathrm{U}$ test for those continuous data not normally distributed (shown as Mean Rank). ${ }^{\dagger}$ YFAS-C food addiction diagnosis: endorsement of $\geq 3$ symptoms + criterion met for clinical impairment or distress. ${ }^{\ddagger}$ Children YFAS-C (25 items). ${ }^{\natural}$ Data were missing for 24 children. ${ }^{6}$ Data were missing for 38 children. ${ }^{\dagger}$ Data were missing for 22 children. $\mho$ Data were missing for 26 children. ${ }^{\S}$ Data were missing for 25 children. ${ }^{\circ}$ Data were missing for 25 children. ${ }^{\epsilon}$ A symptom count without diagnosis, adding up all of the scores for each of the criterion (e.g. tolerance, withdrawal, and use despite negative consequences) without considering the score of clinical significance. This should range from 0 to 7 ( 0 symptom to 7 symptoms). 
Table 3. Comparison of sociodemographic and anthropometric variables between Iranian elementary school students with and without food addiction $\mathrm{A}^{9}$

\begin{tabular}{|c|c|c|c|c|}
\hline Food addiction variables & $\begin{array}{c}\text { Undiagnosed } \\
(\mathrm{n}=172)\end{array}$ & Diagnosed $^{\dagger}(\mathrm{n}=37)$ & $\begin{array}{c}\text { Test statistic } \\
(\chi 2 / \text { Mann-Whitney } U) \\
\end{array}$ & $\mathrm{p}$ \\
\hline \multicolumn{5}{|l|}{ Demographic characteristics } \\
\hline \multicolumn{5}{|l|}{ Sex } \\
\hline Males & $111(82.2)$ & $24(17.8)$ & 0.6 & $>0.05$ \\
\hline Females & $61(83.6)$ & $12(16.4)$ & & \\
\hline Age & 100.7 & 122.7 & 2441 & $<0.05$ \\
\hline \multicolumn{5}{|l|}{ Age category (\%) } \\
\hline$<8 \mathrm{yr}$ & $59(90.8)$ & $6(9.2)$ & 4.3 & $<0.05$ \\
\hline$>8 \mathrm{yr}$ & $113(79)$ & $30(21)$ & & \\
\hline \multicolumn{5}{|l|}{ Socioeconomic status (\%) } \\
\hline \multicolumn{5}{|l|}{ Paternal occupation } \\
\hline Worker, employee, retired & $96(85)$ & $17(15)$ & 1 & $>0.05$ \\
\hline $\begin{array}{l}\text { Practitioner (medical physician, lower, } \\
\text { etc.) and self-employment }\end{array}$ & $70(79.5)$ & $18(20.5)$ & & \\
\hline Maternal occupation & & & & \\
\hline Employed & $28(80)$ & $7(20)$ & 0.13 & $>0.05$ \\
\hline Non-employed (housewife and retired) & $138(82.6)$ & $29(17.4)$ & & \\
\hline Paternal education level & & & & \\
\hline College education & $86(85.1)$ & $15(14.9)$ & 0.94 & $>0.05$ \\
\hline Non-college education (Diploma and less) & $84(80)$ & $21(20)$ & & \\
\hline \multicolumn{5}{|l|}{ Maternal education level } \\
\hline College education & $66(85.7)$ & $11(14.3)$ & 0.9 & $>0.05$ \\
\hline Non-college education (Diploma and less) & $104(80.6)$ & 25 (19.4) & & \\
\hline Anthropometric measurements & & & & $>0.05$ \\
\hline Weight & 101.3 & 117.4 & 2540.5 & $>0.05$ \\
\hline Height & 101 & 119 & 2485 & $>0.05$ \\
\hline Body-mass index (BMI) * & 102.4 & 112.04 & 2728.5 & $>0.05$ \\
\hline BMI Z-score & 103.7 & 105.5 & 2957 & $>0.05$ \\
\hline \multicolumn{5}{|l|}{ BMI z-score category ${ }^{\S}(\%)$} \\
\hline Underweight & $23(76.7)$ & $7(23.3)$ & 2.3 & $>0.05$ \\
\hline Healthy weight & $59(86.8)$ & $9(13.2)$ & & \\
\hline Overweight & $38(86.4)$ & $6(13.6)$ & & \\
\hline Obese & $37(80.4)$ & $9(19.6)$ & & \\
\hline Morbidly obese & 15 (78.9) & $4(21.1)$ & & \\
\hline
\end{tabular}

However, Mann-Whitney U-test indicated no significant differences in weight, height, BMI, BMI z-score, and BMI Z-score categories between students with food addiction diagnosis and those undiagnosed overall and when sorted by sex (Table 3). Moreover, Kruskal-Wallis H test revealed no significant difference in weight, height, BMI, and BMI zscore between the students with different categories of food addiction severity. Also, Chi- square test showed no significant differences between students with and without food

Table 4. Association of demographic variables with food addiction score on continuous version of the YFAS-C in Iranian elementary school students

\begin{tabular}{lccc}
\hline Demographic variables & Mean Rank & Mann-Whitney U & $\mathrm{p}$ \\
\hline Sex & & & $<0.05$ \\
$\quad$ Male & 95.9 & 3036 & \\
$\quad$ Female & 79.7 & & $<0.05$ \\
Age (year) & & 2684 & \\
$\quad<8$ y & 77.2 & & \\
$\quad>8 Y$ & 95.5 & & \\
\hline
\end{tabular}

Table 5. Correlation between food addiction score on the continuous version of the scale and anthropometric indicators among Iranian elementary school students by sex $(\mathrm{n}=222)$ 9

\begin{tabular}{|c|c|c|c|}
\hline Anthropometric measurements & Sex & $\mathrm{R} \neq$ & $\mathrm{p}$ \\
\hline \multirow[t]{3}{*}{ Height $(\mathrm{cm})$} & Males & 0.263 & $<0.01$ \\
\hline & Females & 0.038 & $>0.05$ \\
\hline & Total & 0.2 & $<0.01$ \\
\hline \multirow{3}{*}{ Weight (kg) } & Males & 0.198 & $<0.05$ \\
\hline & Females & 0.267 & $<0.05$ \\
\hline & Total & 0.215 & $<0.01$ \\
\hline \multirow[t]{3}{*}{ Body-mass index (BMI) } & Males & 0.103 & $>0.05$ \\
\hline & Females & 0.354 & $<0.01$ \\
\hline & Total & 0.188 & $<0.05$ \\
\hline \multirow{3}{*}{ BMI z-score } & Males & 0.06 & $>0.05$ \\
\hline & Females & 0.333 & $<0.01$ \\
\hline & Total & 0.157 & $<0.05$ \\
\hline
\end{tabular}

‡ Spearman partial correlation coefficients 
addiction diagnosis and different categories of food addiction severity in BMI $\mathrm{z}$-score category $(\mathrm{p}>0.05)$ (data are displayed in Table 3).

\section{Discussion}

This was the first survey to examine the relationships among food addiction, anthropometric, and sociodemographic indicators in Iranian elementary school students. The results indicated that the food addiction prevalence in a general population of Iranian children and adolescents was $17.3 \%$ and that more than $50 \%$ of food addicted students were diagnosed with severe food addiction. Also, the most common symptoms associated with food addiction were inability to cut down, withdrawal, and tolerance, identifying food addiction as a contributor to their clinical eating problems. Furthermore, those students with food addiction diagnosis were older than undiagnosed ones. Also, students aged over 8 years had a more prevalence of food addiction than those aged under 8 years. Male students showed a higher food addiction score on the continuous version of the YFAS-C than females. Besides, male students aged over 8 years had a higher food addiction score than males aged under 8 years. Moreover, significant positive correlations were observed between all anthropometric indicators and food addiction score in the entire sample; moreover, there was a similar correlation among food addiction score, BMI, and BMI z-score in females. However, weight and height, but not BMI and BMI z-score, had a positive correlation with food addiction score in males.

Few studies have investigated the prevalence of addictive-like eating behavior in children and adolescents $(1,25$, $26,35)$. The prevalence of food addiction in a recent study on American children aged 5 to 12 years was reported to be $22.7 \%$ (1). In a study, Merlo et al. found (26) that $15.2 \%$ of overweight children "usually", "often" or "always" think that they are food addicted. Although the prevalence observed in the current study for food addiction is marginally similar to the mean prevalence of food addiction in adult population [22.2\% (24), ranging between 5.4\% (13) and $56.8 \%$ (19)], with higher values found for overweight or obese women $(13,39,40)$. The prevalence of the current sample is higher than what has been observed in the past from 4 to 16 year old children in the United States with different races/ethnicities including Caucasians, AfricanAmericans, Hispanics, and others (7.2\%) (25). However, the limitation of the mentioned study was that children's dietary preferences are often dictated by parents. Also, in a study among Turkish obese children and adolescents, the frequency of addictive-like behavior turned out to be $71 \%$ (36). These results suggest the necessity of supportive and educational measures to minimize food addiction prevalence in the population of children and adolescents including the precautionary use of the food addiction label in schools' nutrition programs (41). Also, future preventive programs could be applied including teaching the skills for inhibitory control, joint with modifications in the constructed school setting to improve the safety of schools' nutrition programs (42).

In the presents study, the most common symptom associated with food addiction was "inability to cut down", which means that a child or adolescent desires persistently and does a repeated attempt unsuccessfully to quit (43). The present study found that more than $90 \%$ of food addicted students met this criterion. A similar result was observed in a study on 4 to 16-year-old children in the United States, indicating that "inability to cut down" was the most common criterion among participants who met the diagnostic threshold with a rate of $65.3 \%$ (25). Similarly, in a qualitative internet study, "unsuccessful efforts to cut down" was the second prevalent criterion among food addicted children (35), whereas in adults, higher food addiction scores are associated with decreased activation of a neural region implicated in inhibitory control (e.g. lateral orbitofrontal cortex) during food consumption and greater activation in reward-related regions (e.g. striatum) in response to food indications. Thus, when food is present, children with more addictive-like eating may be prone to increase reward responsivity to the food, leading to consume more calories, and this is combined with lower sensitivity to satiety signals and decreased inhibitory control (28). However, more research is needed to understand the cause of the high prevalence of "inability to cut down" among other symptoms of food addiction in children and adolescents.

"Withdrawal" was a second criterion observed in the present study. This symptom means having physical withdrawal symptoms when abstaining from specific foods two or more times per week (44). In the present study, about $80 \%$ of students with food addiction diagnosis represented "withdrawal" criterion. In a study by Gearhardt et al. (25) in the United States, the prevalence of "withdrawal" symptoms among children and adolescents was $18.9 \%$. Also, in the study of Pretlow et al. (35), "withdrawal" was a fifth criterion observed in food addicted children. Eating can be dysregulated by stress, stimulating a favorite for highly palatable (high fat/high sugar) foods (17). Over time, exposure to palatable foods seems to disrupt brain reward function, leading to "withdrawal" symptoms when the specific foods are cut down (4), and encouraging sustained intake (17). Considering a high prevalence of different grades of stress among Iranian adolescents (45) as well as many parallels between stress and feeding behavior leading to withdrawal, it is not surprising that "withdrawal" is the second common food addiction criterion observed in the present study. However, it is necessary to design studies describing psychological characteristics of participants who met the "withdrawal" criterion as a common physiological measure of food addiction among Iranian food addicted children and adolescents besides evaluating the relationship between the "withdrawal" prevalence and the social stimuli inducing stress in Iranian children and adolescents.

"Tolerance" was the third criterion observed in the present study. Progressively eating more and more over time may be characterized as "tolerance" (35). In the present study, "tolerance" criterion was represented in more than $70 \%$ of students with food addiction diagnosis. Similarly, in a previous qualitative internet study, $77 \%$ of respondent children met "tolerance" as "the fourth observed criterion (35). On the contrary, the prevalence of "tolerance" symptom among children and adolescents in the United states 
was reported as nearly $25 \%$ (25). Based on an animal study, eating excessive amounts of fat- and sugar-rich foods may develop "tolerance". In this condition, the individuals crave more and more to satisfy their hunger (46). Accordingly, it is necessary to evaluate the nutritional intake of children and adolescents when they are assessed for addictive- like eating behavior. Moreover, children participating in the present study were at the growth age. This contradicts the fact that eating more over time might well have resulted from growth along with age (14).

In addition, the results of the present study indicated no significant associations between the status of food addiction diagnosis and food addiction severity and socioeconomic status variables including paternal and maternal occupation and education level. However, the students with food addiction diagnosis were older than the undiagnosed. Also, the prevalence of food addiction was higher among students aged over 8 years than those under 8 years, with a higher food addiction score in male students compared with females. Besides, food addiction score was higher in male students aged over 8 years than those under 8. The results of a similar study confirmed the nonsignificant associations between food addiction and sociodemographic variables (1). Moreover, a survey evaluating high-calorie, low-nutrient (HCLN) food/beverage intake in fourth-grade children demonstrated that male sex and low inhibitory control independently predicted HCLN intake (42). Pre- and early adolescents (10-14 years old) gradually develop independence. They commute geographically within (and outside) their home area, especially on the school route. This commute allows children to purchase their own food from convenience and grocery stores located at school routes. Most of the purchases include snacks such as candy, ice cream, and sandwiches (47). Accordingly, the importance of educational interventions targeted at children over 8 years of age with a focus on male students, whose particular food selections are described as almost completely high- calorie, low-nutrient-dense snack foods, is emphasized. Specially, most addictive foods are dense in calorie and high in refined sugars and fats with low nutrients and have exceptional relevance to obesity, which is nowadays a primary and ever-increasing health problem worldwide (42).

The present study found significant positive correlations between all anthropometric indicators and food addiction score in the entire sample. Moreover, a similar correlation was found among food addiction score, BMI, and BMI zscore in females. However, weight and height had a positive correlation with food addiction score in males. A similar study found a positive association between YFAS$\mathrm{C}$ scores based on parental report and BMI z-score and obesity in children (1). Similar results were found in university students (8) and obese and overweight adolescents (36). A recent study showed that children who consume more calories at dinner have scores that indicate a greater level of food addiction. In older children, BMI percentile was significantly associated with total calories consumed after dinner, but not with any other measures of calorie intake (28). A qualitative prospective study in the United States revealed that the efforts of children to lose weight are consistent with their BMI percentile, suggesting that the dependence on the food pleasure may range from partially dependent (addicted) in overweight children to fully dependent (addicted) in obese children; and thus, children with addictive tolerance mode may be morbidly obese (35). These results highlight the importance of considering the food selections and calorie contents of selected foods in early adolescence and suggest the necessity of the preventive intervention of food addiction aiming to decrease the risk of obesity related to addictive-like eating behavior in female students.

One limitation of the current study was the sex disproportion between the number of participants in the study so that the number of male contributors was larger than females, given the possiblity that the real prevalence of food addiction in the general population may be lower than $17.3 \%$ if the study had included an equal number of male and female students. Thus, future studies with equal number of female and male students as participants are suggested. Another study limitation is that parental control over eating behavior was not assessed. Parental behavior may impact the likelihood that children would exhibit addictive-like eating. Understanding the impact of parenting behavior on addictive-like eating behaviors in children is an important future direction. Moreover, to address potential comprehension issues for younger participants, parents were instructed that they could help their children in completing the questionnaire if the child needed assistance. Parental assistance, which is considered as another limitation of this study, may impact the interpretation of the questionnaire; however, information regarding whether parents assisted their child was not considered in data collection.

A possible power of the present study was evaluation of the relationship between sociodemographic indicators with food addiction diagnosis and the frequency of food addiction symptoms for the first time, as well as a narrow age range of the participants ( 7 to 13 years of age), which strengthens the observed relationship between age and food addiction diagnosis. It is necessary to develop longitudinal studies to evaluate the causal relationship between food addiction and childhood and adolescence obesity/ overweight.

There is a need to evaluate the relationship between food addiction criteria prevalence and psychiatric variables in Iranian children and adolescents for future research to better understand addictive-like eating among students in early adolescence. Moreover, longitudinal studies should be conducted to examine whether early patterns of addictive-like eating can predict future problematic eating behaviors.

\section{Conclusion}

The present study expanded the limited literature on food addiction in children and adolescents. The results indicated that food addiction diagnosis along with clinically significant impairment is more prevalent in male students aged over 8 years. Also, it was found that the risk of obesity related to addictive-like eating behavior was higher in female children and adolescents than in males. Results of the pre- 
sent study suggest that food addiction may be a very important problem in childhood and early adolescence contributing to a risk of overweight/obesity in Iranian students. Identification of food addiction may improve obesity treatment efforts for this age/sex group.

\section{Acknowledgments}

The authors would like to thank the employees of Ahvaz organization of education and training as well as the students and parents who contributed to the study.

\section{Financial and Ethical Issues}

This study was supported financially by the office of Vice-Chancellor for Research and Technology, Ahvaz Jundishapur University of Medical Sciences, Ahvaz, Iran (Grant number: 94S119).

\section{Conflict of Interests}

The authors declare that they have no competing interests.

\section{References}

1. Burrows T, Skinner J, Joyner MA, Palmieri J, Vaughan K, Gearhardt AN. Food addiction in children: Associations with obesity, parental food addiction and feeding practices. Eat Behav. 2017;26:114-20.

2. Di Segni M, Patrono E, Patella L, Puglisi-Allegra S, Ventura R. Animal models of compulsive eating behavior. Nutrients. 2014;6(10):4591-609.

3. Avena NM, Rada P, Hoebel BG. Evidence for sugar addiction: behavioral and neurochemical effects of intermittent, excessive sugar intake. Neurosci Biobehav Rev. 2008;32(1):20-39.

4. Johnson PM, Kenny PJ. Dopamine D2 receptors in addiction-like reward dysfunction and compulsive eating in obese rats. Nature Neurosci 2010;13(5):635-41.

5. Pedram P, Sun G. Hormonal and dietary characteristics in obese human subjects with and without food addiction. Nutrients. 2014;7(1):223-38.

6. Gearhardt AN, Yokum S, Orr PT, Stice E, Corbin WR, Brownell KD. Neural correlates of food addiction. Arch Gen Psychiatry. 2011;68(8):808-16.

7. Davis C, Loxton NJ, Levitan RD, Kaplan AS, Carter JC, Kennedy JL. 'Food addiction' and its association with a dopaminergic multilocus genetic profile. Physiol Behav. 2013;118:63-9.

8. Sanlier N, Turkozu D, Toka O. Body Image, Food Addiction, Depression, and Body Mass Index in University Students. Ecol Food Nutr. 2016;55(6):491-507.

9. Burrows T, Hides L, Brown R, Dayas CV, Kay-Lambkin F. Differences in Dietary Preferences, Personality and Mental Health in Australian Adults with and without Food Addiction. Nutrients. 2017;9(3).

10. Murphy CM, Stojek MK, MacKillop J. Interrelationships among impulsive personality traits, food addiction, and Body Mass Index. Appetite. 2014;73:45-50.

11. Meule A, Kubler A. Food cravings in food addiction: the distinct role of positive reinforcement. Eat Behav. 2012;13(3):252-5.

12. Meule A GA. Five years of the Yale Food Addiction Scale: Taking stock and moving forward. Curr Addict Rep. 2014: 1-13.

13. Pedram P, Wadden D, Amini P, Gulliver W, Randell E, Cahill F, et al. Food addiction: its prevalence and significant association with obesity in the general population. PLoS One. 2013;8(9):e74832.

14. Burmeister JM, Hinman N, Koball A, Hoffmann DA, Carels RA. Food addiction in adults seeking weight loss treatment. Implications for psychosocial health and weight loss. Appetite. 2013;60(1):103-10.

15. Brunault P, Ballon N, Gaillard P, Reveillere C, Courtois R. Validation of the French version of the yale food addiction scale: an examination of its factor structure, reliability, and construct validity in a nonclinical sample. Can J Psychiatry. 2014;59(5):276-84.

16. Gearhardt AN, White MA, Masheb RM, Grilo CM. An examination of food addiction in a racially diverse sample of obese patients with binge eating disorder in primary care settings. Compr Psychiatry. 2013;54(5):500-5.

17. Mason SM, Flint AJ, Field AE, Austin SB, Rich-Edwards JW. Abuse victimization in childhood or adolescence and risk of food addiction in adult women. Obesity. 2013;21(12):E775-81.

18. Karandish M, Naghashpour M, Shahbazian H, Latifi SM. Food Addiction Is More Prevalent in Women with Metabolic Syndrome. Intl J Psychol Cog Sci. 2015;1(1):1-6.

19. Gearhardt AN, White MA, Masheb RM, Morgan PT, Crosby RD, Grilo CM. An examination of the food addiction construct in obese patients with binge eating disorder. Int J Eat Disord. 2012;45(5):65763.

20. Meule A, Heckel D, Kubler A. Factor structure and item analysis of the Yale Food Addiction Scale in obese candidates for bariatric surgery. Eur Eat Disord Rev. 2012;20(5):419-22.

21. Davis C, Curtis C, Levitan RD, Carter JC, Kaplan AS, Kennedy JL. Evidence that 'food addiction' is a valid phenotype of obesity. Appetite. 2011;57(3):711-7.

22. Gearhardt AN, Corbin WR, Brownell KD. Preliminary validation of the Yale Food Addiction Scale. Appetite. 2009;52(2):430-6.

23. Flint AJ, Gearhardt AN, Corbin WR, Brownell KD, Field AE, Rimm EB. Food-addiction scale measurement in 2 cohorts of middle-aged and older women. Am J Clin Nutr. 2014;99(3):578-86.

24. Pursey KM, Stanwell P, Gearhardt AN, Collins CE, Burrows TL. The prevalence of food addiction as assessed by the Yale Food Addiction Scale: a systematic review. Nutrients. 2014;6(10):4552-90.

25. Gearhardt AN, Roberto CA, Seamans MJ, Corbin WR, Brownell KD. Preliminary validation of the Yale Food Addiction Scale for children. Eat Behav. 2013;14(4):508-12.

26. Merlo LJ, Klingman C, Malasanos TH, Silverstein JH. Exploration of food addiction in pediatric patients: A preliminary investigation. J Addict Med. 2009;3(1):26-32.

27. Lisdahl KM GE, Wright NE, Shollenbarger S. Dare to delay? The impacts of adolescent alcohol and marijuana use onset on cognition, brain structure, and function. Front Psychiat. 2013;4.

28. Richmond RL, Roberto CA, Gearhardt AN. The association of addictive-like eating with food intake in children. Appetite. 2017;117:82-90.

29. Schulte EM, Tuttle HM, Gearhardt AN. Belief in Food Addiction and Obesity-Related Policy Support. PLoS One. 2016;11(1):e0147557.

30. Eichen DM, Lent MR, Goldbacher E, Foster GD. Exploration of "food addiction" in overweight and obese treatment-seeking adults. Appetite. 2013;67:22-4.

31. Pursey KM, Gearhardt AN, Burrows TL. The relationship between "food addiction" and visceral adiposity in young females. Physiol Behav. 2016;157:9-12.

32. Johnston LD OMP, Bachman JG, Schulenberg JE. Monitoring the future national results on adolescent drug use: Overview of key findings, 2011. Institute for Social Research: The University of Michigan; 2012.

33. Nickelson J, Lawrence JC, Parton JM, Knowlden AP, McDermott RJ. What proportion of preschool-aged children consume sweetened beverages? J Sch Health. 2014;84(3):185-94.

34. Pan L, Li R, Park S, Galuska DA, Sherry B, Freedman DS. A longitudinal analysis of sugar-sweetened beverage intake in infancy and obesity at 6 years. Pediatrics. 2014;134 Suppl 1:S29-35.

35. Pretlow RA. Addiction to highly pleasurable food as a cause of the childhood obesity epidemic: a qualitative Internet study. Eat Disord. 2011;19(4):295-307.

36. Keser A, Yuksel A, Yesiltepe-Mutlu G, Bayhan A, Ozsu E, Hatun S. A new insight into food addiction in childhood obesity. Turk J Pediatr. 2015;57(3):219-24

37. BMI classification [Internet]. 2015 [cited 23 July 2015].

38. Association AP. Diagnostic and statistical manual of mental disorders. 4th ed. Washington DC 2000.

39. Pursey KM, Collins CE, Stanwell P, Burrows TL. The stability of 'food addiction' as assessed by the Yale Food Addiction Scale in a nonclinical population over 18-months. Appetite. 2016;96:533-8.

40. Meule A GA. Five years of the Yale Food Addiction Scale. Taking stock and moving forward. Curr Addict Rep. 2014;1:193-205.

41. Cullen AJ, Barnett A, Komesaroff PA, Brown W, O'Brien KS, Hall $\mathrm{W}$, et al. A qualitative study of overweight and obese Australians' views of food addiction. Appetite. 2017.

42. Pentz MA, Spruijt-Metz D, Chou CP, Riggs NR. High calorie, low nutrient food/beverage intake and video gaming in children as potential signals for addictive behavior. Intl $\mathrm{J}$ Environ Res Pub Health. 2011;8(12):4406-24.

43. Meule A GA. Five years of the Yale Food Addiction Scale: Taking 
Food addiction in children

stock and moving forward. Curr Addict Rep. 2014;1:193-205.

44. Parylak SL, Koob GF, Zorrilla EP. The dark side of food addiction. Physiol Behav. 2011;104(1):149-56.

45. Ghofranipour F, Saffari M, Mahmoudi M, Montazeri A. Demographical and psychological determinants of depression, among a sample of Iranian male adolescents. Intl J Prevent Med. 2013;4(10): 1217-23.

46. Avena NM, Rada P, Hoebel BG. Underweight rats have enhanced dopamine release and blunted acetylcholine response in the nucleus accumbens while bingeing on sucrose. Neuroscience. 2008;156(4):86571.

47. Engler-Stringer R, Schaefer J, Ridalls T. An examination of the roles played by early adolescent children in interactions with their local food environment. Can J Public Health. 2016;107(Suppl 1):5296. 